\title{
Identification of damage in a beam structure by using mode shape curvature squares
}

\author{
S. Rucevskis* and M. Wesolowski \\ Institute of Materials and Structures, Riga Technical University, Kalku Str. 1, LV-1658, Riga, Latvia
}

\begin{abstract}
During the last decades a great variety of methods have been proposed for damage detection by using the dynamic structure characteristics, however, most of them require modal data of the structure for the healthy state as a reference. In this paper the applicability of the mode shape curvature squares determined from only the damaged state of the structure for damage detection in a beam structure is studied. To establish the method, two aluminium beams containing different-size mill-cut damage at different locations are tested by using the experimentally measured modal data. The experimental modal frequencies and the corresponding mode shapes are obtained by using a scanning laser vibrometer with a PZT actuator. From the mode shapes, mode shape curvatures are obtained by using a central difference approximation. With the example of the beams with free-free and clamped boundary conditions, it is shown that the mode shape curvature squares can be used to detect damage in the structures. Further, the extent of a mill-cut damage is identified via modal frequencies by using a mixed numerical-experimental technique. The method is based on the minimization of the discrepancy between the numerically calculated and experimentally measured frequencies.
\end{abstract}

Keywords: Damage detection, dynamic response, mode shape curvature, scanning laser vibrometer

\section{Introduction}

Structural health monitoring and damage detection in civil, mechanical and aerospace engineering constructions has become one of the most important keys in maintaining the integrity and safety of a structure. During the last decades vibration-based damage detection methods have been attracting most attention due to their simplicity of implementation. These methods are based on the fact that dynamic characteristics, i.e., the modal frequencies, mode shapes, and modal damping are directly related to the stiffness of the structure. Therefore, a change in natural frequencies or a change in mode shapes will indicate a loss of the stiffness. Valuable reviews of the state of the art in the methods for detecting, localizing, and characterizing damage by examining the changes in the measured vibration parameters can be found in [1,2]. Many studies have investigated the effects of damage on mode shapes [3-5] and corresponding mode shape curvatures [6-8]. These papers show that mode shape curvatures are highly sensitive to damage and can be used to localize it. However, the major drawback of those methods is a need for the data of the healthy structure which sometimes could be difficult to obtain or even impossible. To overcome this issue Gapped Smoothing Techniques [9-11] were introduced which allow the damage detection in a structure without prior knowledge on the healthy state. The basic idea of the methods is that the mode shape curvature of the healthy structure has a smooth surface, and it can be approximated by a polynomial. The square of the difference between the measured curvature and the smoothed polynomial is defined as damage index and maximum value indicates the location and size of the damage.

In this paper, a method which uses mode shape curvature squares for the damage detection in a beam determined from only the damaged state of the structure is described and compared with other relevant damage detection methods referenced in literature. The proposed method is based on the idea that damage induced reduction of the flexural

\footnotetext{
${ }^{*}$ Corresponding author. E-mail: sandris_r@ $@$ bf.rtu.lv.
} 
stiffness of the structure subsequently causes an increase in the magnitude of the mode shape curvature square and thus the mode shape curvature square may be considered an indicator for the damage location. Compared to existing mode shape damage detection methods, the advantage of the proposed method is that it requires mode shape information only from the damaged state of the structure and can give reliable results in a simpler way. Experimental modal frequencies and corresponding mode shapes obtained by using a scanning laser vibrometer with a PZT actuator are used for illustration of the proposed method. In addition damage extent is identified via the modal frequencies by using a mixed numerical-experimental technique.

\section{Damage detection algorithms}

Since the mode shape curvature squares are derived from mode shapes and also for a better illustration of the proposed method, it was decided to compare the present method with other relevant damage detection methods which employ mode shape information.

\subsection{Mode shape (MS) damage index}

The simplest one is the mode shape damage index. It represents the difference between the mode shapes of the healthy and the damaged structures [3]

$$
\Delta v_{i}=\left|v_{i}^{d}-v_{i}\right|
$$

where $v_{i}^{d}$ and $v_{i}$ are mode shapes of the damaged and the healthy state of a structure, respectively, and $i$ denotes the node number or measured point.

The experimentally measured mode shapes are inevitably corrupted by measurement noise. This noise introduces local perturbations into the mode shape which can lead to peaks in the mode shape slope, curvature and curvature square profiles. These peaks could be mistakenly interpreted as damage or they could mask the peaks induced by real damage in a beam and lead to false or missed detection of damage. To overcome this problem, it is proposed to average the sum of damage indices from each mode. To summarize the results for all modes, the index is proposed as

$$
M S_{i}=\frac{1}{N} \sum_{n=1}^{N}\left(\Delta v_{i}\right)_{n}
$$

where $N$ is the total number of modes to be considered.

\subsection{Mode shape slope (MSS) damage index}

This algorithm uses the change in the mode shape slope

$$
\Delta v_{i}^{\prime}=\left|v_{i}^{\prime}-v_{i}^{\prime}\right|
$$

The central difference approximation is used to derive the mode shape slope from the mode shape

$$
v_{i}^{\prime}=\frac{\left(v_{i+1}-v_{i-1}\right)}{2 h}
$$

where $h$ is the distance between two successive nodes or measured points.

If more than one mode is used, the index is given by

$$
M S S_{i}=\frac{1}{N} \sum_{n=1}^{N}\left(\Delta v_{i}^{\prime}\right)_{n}
$$




\subsection{Mode shape curvature (MSC) damage index}

In this algorithm the location of damage is assessed by the difference in the mode shape curvature between the healthy and the damaged case [6]

$$
\Delta v_{i}^{\prime \prime}=\left|v_{i}^{\prime \prime}-v_{i}^{\prime \prime}\right|
$$

The mode shape curvatures are computed from experimentally measured or numerically calculated mode shapes using the central difference approximation

$$
v_{i}^{\prime \prime}=\frac{\left(v_{i+1}-2 v_{i}+v_{i-1}\right)}{h^{2}}
$$

The sum of the damage indices from each mode is defined by

$$
M S C_{i}=\frac{1}{N} \sum_{n=1}^{N}\left(\Delta v_{i}^{\prime \prime}\right)_{n}
$$

\subsection{Mode shape curvature square (MSCS) damage index}

This damage index is defined by [3]

$$
\Delta v_{i}^{\prime \prime 2}=\left|v_{i}^{\prime \prime} d 2-v_{i}^{\prime \prime 2}\right|
$$

For more than one mode used, the index is

$$
M S C S_{i}=\frac{1}{N} \sum_{n=1}^{N}\left(\Delta v_{i}^{\prime \prime 2}\right)_{n}
$$

All the aforementioned methods assess the location of the damage by the largest computed absolute difference between the mode shape function of the damaged and the healthy state of a structure. However, the major drawback of those methods is a need for the data of the healthy structure, which sometimes could be difficult or even impossible to obtain. To overcome this issue it was proposed to use the mode shape curvature squares from only the damaged state of the beam as a damage index.

\subsection{Mode shape curvature square magnitude (MSCSM) damage index}

The vibration strain energy $\left(U_{i}\right)$ associated with the particular mode shape at a point is given by

$$
U_{i}=\frac{1}{2} \int_{x} E I\left(v_{i}^{\prime \prime}\right)^{2} d x
$$

where $v_{i}^{\prime \prime}$ is the mode shape curvature and EI is the flexural stiffness of the structure. The idea of the proposed method is based on the relationship between the mode shape curvature square and the flexural stiffness of a structure. Damage induced reduction of the flexural stiffness of the structure subsequently causes an increase in the magnitude of the mode shape curvature square. The increase in the magnitude of the curvature square is local in nature, thus the mode shape curvature square may be considered an indicator for the damage location. The location of the damage is assessed by the largest magnitude of the mode shape curvature square. The summarized damage index for all modes is proposed as

$$
\operatorname{MSCSM} M_{i}=\frac{1}{N} \sum_{n=1}^{N}\left(v_{i}^{\prime \prime} d 2\right)_{n}
$$




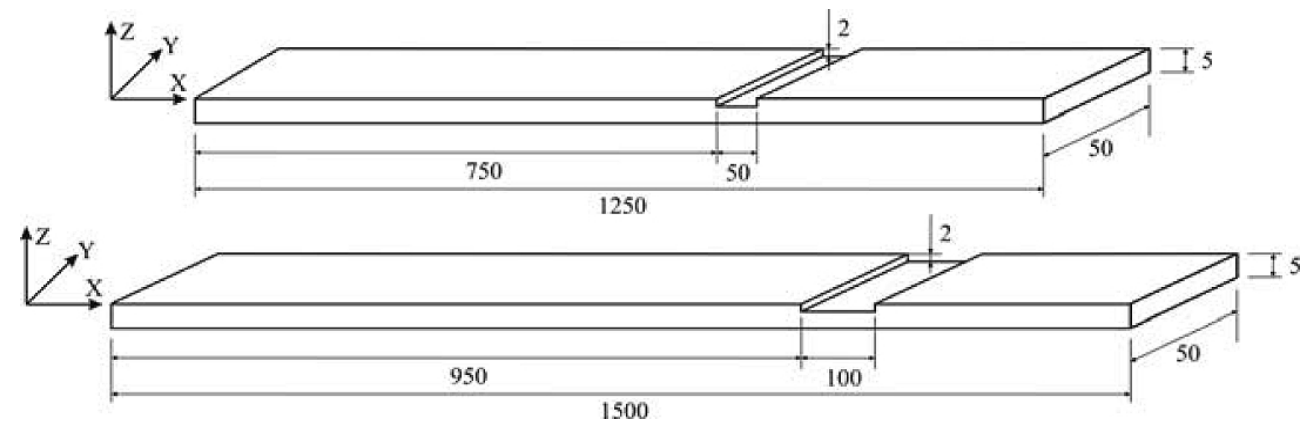

Fig. 1. Geometry and dimensions of the test beams containing mill-cut damage.

\section{Numerical analysis}

To verify the validity and effectiveness of the damage algorithms introduced above, numerical modal analysis based on the finite element (FE) method was performed. Numerical analysis was carried out by using the commercial FE software ANSYS.

Two aluminium beams containing different size mill-cut damage at different locations are considered in this study. The geometrical configuration of the beams is shown in Fig. 1. The dimensions of Beam 1 are as follows: length L $=1250 \mathrm{~mm}$, width $\mathrm{B}=50 \mathrm{~mm}$, and thickness $\mathrm{H}=5 \mathrm{~mm}$. Mill-cut damage with a depth of $2 \mathrm{~mm}$ and size (width) of $50 \mathrm{~mm}$ is introduced at a distance of $750 \mathrm{~mm}$ from one edge of the beam. The dimensions of Beam 2 are $1500 \times$ $50 \times 5 \mathrm{~mm}$. Damage with a depth of $2 \mathrm{~mm}$ and size (width) of $100 \mathrm{~mm}$ is introduced at a distance of $950 \mathrm{~mm}$.

Experimentally determined material properties are: Young's modulus $\mathrm{E}=69 \mathrm{GPa}$, Poission ratio $\nu=0.31$ and mass density $\rho=2708 \mathrm{~kg} / \mathrm{m}^{3}$. Finite element models for beams consist of two dimensional beam elements (ANSYS 11.0). Each node has three degrees of freedom, namely translations along the $\mathrm{X}$ and $\mathrm{Y}$ axes and rotation along the $\mathrm{Z}$ axis. Finite element length of $10 \mathrm{~mm}$ is considered: thus Beam 1 is constructed by means of 125 equal length elements $(i=126$ nodes) and Beam 2 is constructed by means of 150 elements $(i=151$ nodes $)$. For the healthy beam, a constant stiffness $E I$ is assumed for all elements, while the damaged beam is modelled by reducing the stiffness of the selected elements. Reduction of stiffness is achieved by decreasing the thickness of elements in the damaged region of the beam, which consequently reduces the moment of inertia $I$. The modal frequencies and corresponding mode shapes for the first 15 flexural modes of both the healthy and the damaged beams were calculated.

\section{Experimental set-up}

Modal frequencies of the test beams are measured by the POLYTEC PSV-400-B scanning laser vibrometer (SLV). General experiment set-up consists of the PSV-I-400 LR optical scanning head equipped with the highly sensitivity vibrometer sensor (OFV-505), OFV-5000 controller, PSV-E-400 junction box, the amplifier Bruel\&Kjaer type 2732, and the computer system with data acquisition board and PSV Software (Fig. 2). The system requires defining the geometry of the object and setting up a scanning grid. To match the finite element model 126 equally spaced scanning points are distributed to cover Beam 1 along its length and 151 scanning points are set for Beam 2. The free-free (all edges free) boundary conditions are simulated during the experiment by suspending the beam with two thin threads. In order to simulate the clamped-clamped (two ends fixed) boundary conditions experimentally, two vices are used to fix the ends of the beam $(10 \mathrm{~mm})$ with the clamped torque equal to $20 \mathrm{Nm}$. The beam is then been excited by an input periodic chirp signal generated by the internal generator with a $1600 \mathrm{~Hz}$ bandwidth through a piezoelectric actuator (PZT). The excitation with small piezoelectric discs works via the radial expansion of the disc causing a bending moment to the beam surface. As a result of this excitation the beam starts to vibrate within the frequency band of the input signal. After the measurement is performed at one point, the vibrometer automatically moves the laser beam to another point of the scan grid, measures the response using the Doppler principle and validates the measurement with the signal-to-noise ratio. The procedure is repeated until all scan points have been measured. The modal frequencies and corresponding mode shapes are obtained by taking the Fast Fourier Transform of the response signal. 


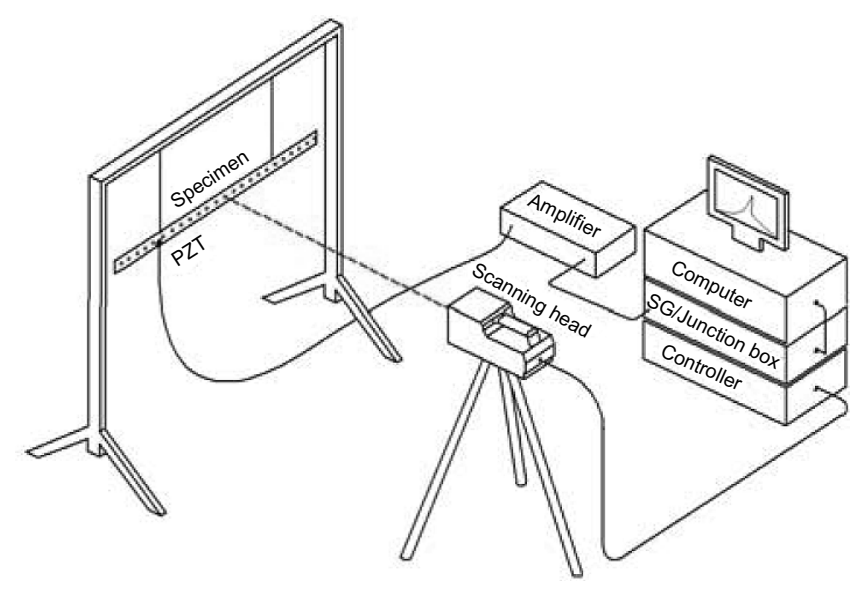

Fig. 2. Scheme of experimental set-up.

\section{Results of damage detection}

Results of the mode shape based damage detection methods are given in Figs 3 and 4. For comparison purposes the damage indexes are also calculated by employing the mode shape information obtained via the finite element simulations. In Figs 3 and 4 the introduced location and size of the damage is indicated by grey color. From the results presented in Fig. 3 it is seen that neither MS nor MSS damage indexes obtained from both the numerically calculated and experimentally measured mode shape information can reliably detect the location and size of the damage. MSC and MSCS damage index methods as well as the proposed MSCSM damage index method succeeded in pointing out the damage size and location. From the damage indexes obtained by using the numerically calculated mode shape information it is seen that the largest peak value appears at the location of the damage demonstrating that the proposed damage index methods can successfully detect and locate the damage. When the experimentally measured mode shape information is employed, a large peak is observed at the location of the damage, however, the largest peaks appear at the boundaries of the beam which can be mistakenly interpreted as damage. This could be explained by the free-free (FF) boundary conditions used in the experiment. In order to minimize the effect of the boundary conditions on damage detection indexes, it was decided to test the beams with the clamped-clamped (CL) boundary conditions. Again, two out of four methods included in the investigation for comparison purposes as well as the proposed MSCSM damage index method were capable of indicating the location and size of the damage. MSCS and MSCSM damage index plots for the beams with the CL boundary conditions are presented in Fig. 4 . One can see that the boundary effect has been reduced significantly and this time the largest peaks in the MSCSM damage index plot are seen at the location of the damage. As seen form the damage index plots which successfully pointed out the location of the damage, the largest peeks are located at the boundaries of the introduced damage region indicated in grey color and thus these peaks can be considered for estimation of the size of the damage. The size of the damage is estimated between two largest peeks of the MSCSM damage index.

\section{Identification of damage extent}

Employing the proposed damage detection method the location and size of the damage was correctly found. Once the location and size of the damage was detected, the following interest was to identify damage extent or in this case the depth of damage. The extent of the damage was identified via modal frequencies by using a mixed numerical-experimental technique. The method is based on the minimization of the discrepancy between the numerically calculated and experimentally measured frequencies. For this the first 10 flexural frequencies of the beams with the free-free boundary conditions were used. The free-free boundary conditions were selected because of the best correlation between the numerically calculated and experimentally measured modal frequencies. In Table 1 modal frequencies for the first 10 flexural modes for both the healthy and damaged state of the beams with 

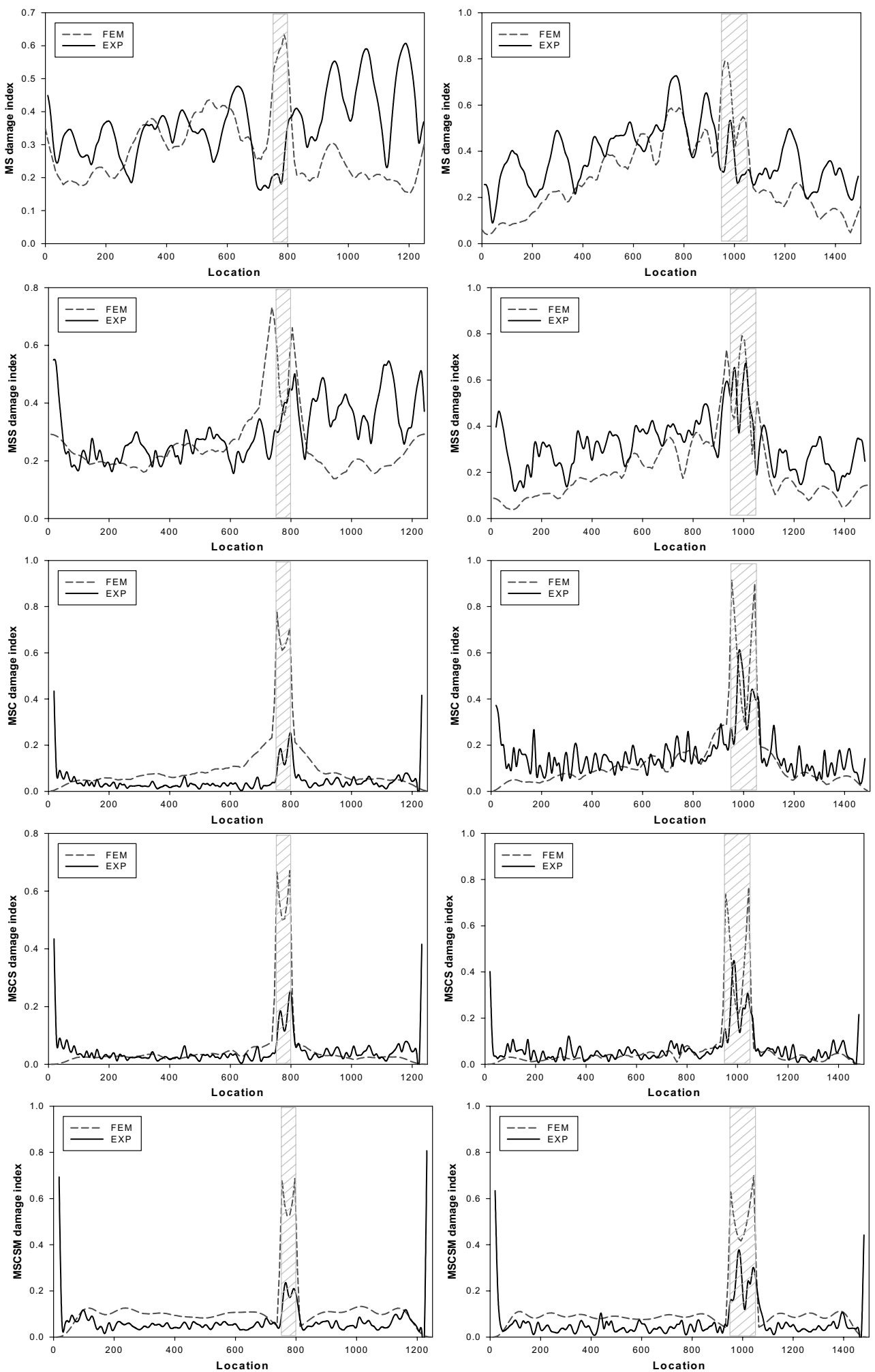

Fig. 3. Damage detection methods for beams with FF boundary conditions; Beam1 - left; Beam 2 - right. 



Fig. 4. Damage detection methods for beams with CL boundary conditions; Beam1 - left; Beam 2 - right.

the free-free boundary conditions, are listed. Residuals characterizing the differences between experimental and numerical frequencies were calculated by the expression

$$
\Delta_{i}=\frac{\left|\omega_{i}^{F E M}-\omega_{i}^{E X P}\right|}{\omega_{i}^{E X P}} \times 100
$$

where $\omega_{i}^{F E M}$ and $\omega_{i}^{E X P}$ are numerically calculated and experimentally measured modal frequencies and $i$ denotes mode number. One can see that residuals between the numerical and experimental frequencies for the healthy beams are very small, which indicate that the finite element model was constructed correctly. On the other hand, frequency residuals for the damaged beams are significantly larger, which indicates that the finite element model has some imperfections, for example, damage representation may not be correct. The damage depth in the beams was modelled by reducing the thickness $\left(h_{1}=3 \mathrm{~mm}\right)$ of the selected elements. Since the damage in the beams was introduced manually by means of a mill, the accuracy of the damage depth could be guaranteed only to the certain limit.

It is assumed that the damage size and location have been set correctly and thus thickness $h_{1}$ of the damage elements is selected as the parameter to be identified. The domain of interest for thickness was selected as follows

$$
2.7 \leqslant h_{1} \leqslant 3.3 \mathrm{~mm}
$$

An accuracy step of $0.1 \mathrm{~mm}$ was selected and the finite element calculations in this domain were performed. Then employing the response surface approach the obtained data were used to build the approximating functions (second order polynomial functions) for all 10 flexural frequencies. These approximating functions represent the relationship between the modal frequencies $\omega_{i}$ and thickness $h_{1}$ of the damage elements. For the identification of thickness two identification functionals were proposed. The first one uses modal frequencies from both the healthy and the damaged states of the beam and is defined by 
Table 1

Flexural frequencies and residuals for the Beam 1 and the Beam 2 with FF boundary conditions

\begin{tabular}{|c|c|c|c|c|c|c|c|c|c|c|c|c|}
\hline \multirow{3}{*}{ Mode } & \multicolumn{6}{|c|}{ Beam 1} & \multicolumn{6}{|c|}{ Beam 2} \\
\hline & \multicolumn{3}{|c|}{ Healthy } & \multicolumn{3}{|c|}{ Damaged } & \multicolumn{3}{|c|}{ Healthy } & \multicolumn{3}{|c|}{ Damaged } \\
\hline & $\begin{array}{c}\omega_{i \underset{E X P}{ } P^{h}} \\
(\mathrm{~Hz})\end{array}$ & $\begin{array}{c}\omega_{i F E M^{h}} \\
(\mathrm{~Hz})\end{array}$ & $\overline{\Delta_{i}(\%)}$ & $\begin{array}{c}\omega_{i E X P}^{d} \\
(\mathrm{~Hz})\end{array}$ & $\begin{array}{c}\omega_{i F E M}^{d} \\
(\mathrm{~Hz}) \\
\end{array}$ & $\overline{\Delta_{i}(\%)}$ & $\begin{array}{c}\omega_{i E X P}^{h} \\
(\mathrm{~Hz})\end{array}$ & $\begin{array}{c}\omega_{i F E M}^{h} \\
(\mathrm{~Hz})\end{array}$ & $\overline{\Delta_{i}(\%)}$ & $\begin{array}{c}\omega_{i E X P}^{d} \\
(\mathrm{~Hz})\end{array}$ & $\begin{array}{c}\omega_{i F E M}^{d} \\
(\mathrm{~Hz})\end{array}$ & $\Delta_{i}(\%)$ \\
\hline 1 & 16.50 & 16.60 & 0.61 & 14.25 & 14.76 & 3.45 & 11.50 & 11.53 & 0.25 & 9.75 & 9.91 & 1.61 \\
\hline 2 & 45.50 & 45.76 & 0.56 & 42.25 & 43.03 & 1.82 & 31.75 & 31.78 & 0.09 & 28.00 & 28.12 & 0.42 \\
\hline 3 & 89.25 & 89.70 & 0.50 & 88.50 & 89.15 & 0.73 & 62.00 & 62.29 & 0.47 & 61.00 & 61.30 & 0.49 \\
\hline 4 & 147.50 & 148.27 & 0.52 & 136.75 & 138.78 & 1.46 & 102.50 & 102.97 & 0.46 & 97.75 & 98.15 & 0.41 \\
\hline 5 & 220.50 & 221.47 & 0.44 & 216.25 & 217.82 & 0.72 & 153.25 & 153.81 & 0.37 & 144.50 & 145.01 & 0.35 \\
\hline 6 & 308.00 & 309.30 & 0.42 & 299.00 & 301.15 & 0.71 & 214.00 & 214.82 & 0.38 & 206.25 & 208.77 & 1.21 \\
\hline 7 & 409.50 & 411.75 & 0.55 & 391.00 & 394.98 & 1.01 & 284.75 & 285.98 & 0.43 & 272.25 & 274.53 & 0.83 \\
\hline 8 & 526.50 & 528.80 & 0.44 & 519.25 & 523.01 & 0.72 & 366.00 & 367.29 & 0.35 & 351.75 & 353.14 & 0.39 \\
\hline 9 & 659.00 & 660.46 & 0.22 & 635.00 & 638.42 & 0.54 & 457.75 & 458.75 & 0.22 & 433.00 & 438.24 & 1.20 \\
\hline 10 & 806.25 & 806.71 & 0.06 & 784.25 & 787.38 & 0.40 & 559.50 & 560.36 & 0.15 & 537.50 & 539.47 & 0.36 \\
\hline Aver. & & & 0.43 & & & 1.16 & & & 0.32 & & & 0.73 \\
\hline
\end{tabular}

$$
\Phi_{1}\left(h_{1}\right)=\sum_{i=1}^{I}\left[\frac{\left(\omega_{i F E M}^{h} \frac{\omega_{i E X P}^{d}}{\omega_{i E X P}^{h}}\right)^{2}-\left(\omega_{i F E M}^{d}\left(h_{1}\right)\right)^{2}}{\left(\omega_{i F E M}^{h} \frac{\omega_{i E X P}^{d}}{\omega_{i E X P}^{u}}\right)^{2}}\right]^{2} ; i=1,2, \ldots, I
$$

where $\omega_{i E X P}^{h}$ and $\omega_{i E X P}^{d}$ are the experimentally measured modal frequencies of the healthy and the damaged states of the beams, respectively. $\omega_{i F E M}^{h}$ are numerically calculated modal frequencies of the healthy state of the beams and $\omega_{i F E M}^{d}\left(h_{1}\right)$ are approximating functions representing the relationship between the modal frequencies and thickness of the damage elements. $I$ is the number of frequencies used in the functional. The idea of this functional is based on the assumption that the numerical frequency ratio $\omega_{i F E M}^{h} / \omega_{i F E M}^{d}$ should be close to the experimental one $\omega_{i E X P}^{h} / \omega_{i E X P}^{d}$.

The second proposed identification functional uses modal frequencies only from the damaged state of the beam and is given as

$$
\Phi_{2}\left(h_{1}\right)=\sum_{i=1}^{I}\left(\frac{\omega_{i E X P}^{d}{ }^{2}-\left(\omega_{i F E M}^{d}\left(h_{1}\right)\right)^{2}}{\omega_{i E X P}^{d}{ }^{2}}\right)^{2} ; i=1,2, \ldots, I
$$

The extent of damage is obtained by minimizing the identification functional $\Phi\left(h_{1}\right)$ subjected to the lower $h_{1}^{\text {min }}$ and upper $h_{1}^{\max }$ bounds of the identification parameter. By minimizing the first identification functional (15), the following thickness of the damage elements were obtained: for Beam $1-h_{1}=2.81 \mathrm{~mm}$, for Beam $2-h_{1}=$ $2.93 \mathrm{~mm}$. Employing the second functional (16): for the Beam $1-h_{1}=2.76 \mathrm{~mm}$, for the Beam $2-h_{1}=2.89$ $\mathrm{mm}$. Now, when the thickness of the damage elements was obtained, it was of interest to evaluate the accuracy of the identification. Verification of the obtained results was performed by numerically calculating modal frequencies in the point of optimum (using the identified thickness of the damage elements).

According to the results given in Tables 2 and 3, the average frequency residuals for the damaged beams are considerably smaller compared to the average residuals when nominal thickness of the damage elements is employed. The residuals for the damaged beams do not exceed $1 \%$ and a good agreement between the average frequency residuals of the healthy and the damaged beams is observed. From this it can be concluded that both identification functionals were capable of identify the extent of damage, the second one showing slightly better results (the average residuals for the damaged beams are smaller). This suggests that the location, size and extent of damage in the beam structure can be obtained without prior knowledge of the healthy state of the structure.

\section{Conclusions}

The present study focuses on the identification of the location, size and extent of mill-cut damage in a beam structure by extracting dynamic characteristics obtained from vibration experiments. It is proposed to use the magnitude of 
Table 2

Flexural frequencies and residuals for the Beam 1 calculated using identified damage extent

\begin{tabular}{|c|c|c|c|c|c|c|c|}
\hline \multirow[t]{3}{*}{ Mode } & \multicolumn{7}{|c|}{ Beam 1} \\
\hline & \multirow[b]{2}{*}{$\begin{array}{l}\omega_{i \underset{E X P}{d}}^{d} \\
\quad(\mathrm{~Hz})\end{array}$} & \multicolumn{2}{|c|}{$h_{1}=3 \mathrm{~mm}$} & \multicolumn{2}{|c|}{$\begin{array}{c}\Phi_{1}\left(h_{1}\right) \\
h_{1}=2.81 \mathrm{~mm}\end{array}$} & \multicolumn{2}{|c|}{$\begin{array}{c}\Phi_{2}\left(h_{1}\right) \\
h_{1}=2.76 \mathrm{~mm}\end{array}$} \\
\hline & & $\omega_{i F \underset{(\mathrm{Hz})}{E M}\left(h_{1}\right)}$ & $\Delta_{i}(\%)$ & $\omega_{i F \underset{(\mathrm{Hz})}{E M}\left(h_{1}\right)}\left(h^{d}\right.$ & $\Delta_{i}(\%)$ & $\begin{array}{c}\omega_{i F \underset{(H z)}{E M}}^{d}\left(h_{1}\right) \\
(\mathrm{Hz})\end{array}$ & $\Delta_{i}(\%)$ \\
\hline 1 & 14.25 & 14.76 & 3.45 & 14.33 & 0.58 & 14.21 & 0.30 \\
\hline 2 & 42.25 & 43.03 & 1.82 & 42.52 & 0.64 & 42.38 & 0.31 \\
\hline 3 & 88.50 & 89.15 & 0.73 & 89.02 & 0.59 & 88.99 & 0.55 \\
\hline 4 & 136.75 & 138.78 & 1.46 & 137.29 & 0.39 & 136.88 & 0.10 \\
\hline 5 & 216.25 & 217.82 & 0.72 & 217.21 & 0.44 & 217.04 & 0.36 \\
\hline 6 & 299.00 & 301.15 & 0.71 & 299.87 & 0.29 & 299.52 & 0.17 \\
\hline 7 & 391.00 & 394.98 & 1.01 & 393.11 & 0.54 & 392.63 & 0.42 \\
\hline 8 & 519.25 & 523.01 & 0.72 & 521.32 & 0.40 & 520.79 & 0.30 \\
\hline 9 & 635.00 & 638.42 & 0.54 & 636.16 & 0.18 & 635.58 & 0.09 \\
\hline 10 & 784.25 & 787.38 & 0.40 & 784.57 & 0.04 & 783.76 & 0.06 \\
\hline Aver. & & & 1.16 & & 0.41 & & 0.27 \\
\hline
\end{tabular}

Table 3

Flexural frequencies and residuals for the Beam 2 calculated using identified damage extent

\begin{tabular}{|c|c|c|c|c|c|c|c|}
\hline \multirow[t]{3}{*}{ Mode } & \multicolumn{7}{|c|}{ Beam 2} \\
\hline & \multirow[b]{2}{*}{$\begin{array}{l}\omega_{i E X P}^{d} \underset{E X}{\quad(\mathrm{~Hz})} \\
\quad\end{array}$} & \multicolumn{2}{|c|}{$h_{1}=3 \mathrm{~mm}$} & \multicolumn{2}{|c|}{$\begin{array}{c}\Phi_{1}\left(h_{1}\right) \\
h_{1}=2.93 \mathrm{~mm}\end{array}$} & \multicolumn{2}{|c|}{$\begin{array}{c}\Phi_{2}\left(h_{1}\right) \\
h_{1}=2.89 \mathrm{~mm}\end{array}$} \\
\hline & & $\omega_{i F \underset{(\mathrm{Hz})}{E(})}\left(h_{1}\right)$ & $\Delta_{i}(\%)$ & 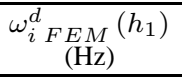 & $\Delta_{i}(\%)$ & 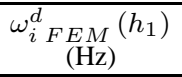 & $\Delta_{i}(\%)$ \\
\hline 1 & 9.75 & 9.91 & 1.61 & 9.79 & 0.36 & 9.71 & 0.40 \\
\hline 2 & 28.00 & 28.12 & 0.42 & 27.92 & 0.29 & 27.81 & 0.70 \\
\hline 3 & 61.00 & 61.30 & 0.49 & 61.23 & 0.37 & 61.18 & 0.30 \\
\hline 4 & 97.75 & 98.15 & 0.41 & 97.90 & 0.16 & 97.76 & 0.01 \\
\hline 5 & 144.50 & 145.01 & 0.35 & 144.69 & 0.13 & 144.52 & 0.01 \\
\hline 6 & 206.25 & 208.77 & 1.21 & 208.24 & 0.96 & 207.91 & 0.80 \\
\hline 7 & 272.25 & 274.53 & 0.83 & 273.95 & 0.62 & 273.62 & 0.50 \\
\hline 8 & 351.75 & 353.14 & 0.39 & 352.72 & 0.27 & 352.48 & 0.21 \\
\hline 9 & 433.00 & 438.24 & 1.20 & 436.54 & 0.81 & 435.52 & 0.58 \\
\hline 10 & 537.50 & 539.47 & 0.36 & 538.48 & 0.18 & 537.92 & 0.08 \\
\hline Aver. & & & 0.73 & & 0.42 & & 0.36 \\
\hline
\end{tabular}

the mode shape curvature square for the detection of the location and size of damage. Compared to existing damage detection methods such as MSC and MSCS damage index methods, the advantage of the proposed method is that it requires mode shape information only from the damaged state of the structure and can give reliable results in a simpler way. In order to reduce the influence of measurement noise on the damage detection from the experimentally measured mode shape information it is proposed to use the average sum of the mode shape curvature squares for all modes. Effectiveness and robustness of the present method is demonstrated by two aluminium beams containing different size mill-cut damage at different locations. It can be concluded that the clamped-clamped instead of the free-free boundary conditions for the beam structure is recommended for the detection of the location and size of damage. The extent of mill-cut damage is identified via modal frequencies by using a mixed numerical-experimental technique. The proposed method is based on the minimization of the discrepancy between the numerically calculated and experimentally measured frequencies. Obtained results show that the thickness of the beam in the damaged region differs from the originally set nominal value, which is explained by the fact that the mill-cut damage in the beams was introduced manually by means of a mill.

\section{Acknowledgements}

This work was partially supported by the European Commission under Framework Program 6, project MOMENTUM, Contract No. MRTN-CT-2005-019198. 


\section{References}

[1] S.W. Doebling, C.R. Farrar, M.B. Prime and D.W. Shevitz, Damage Identification and Health Monitoring of Structural and Mechanical Systems From Changes in Their Vibration Characteristics: A Literature Review, Los Alamos National Laboratory Report LA-13070-MS, 1996.

[2] Y. Xia, Condition Assessment of Structures Using Dynamic Data, PhD. thesis, Nanyang Technology University, Singapore, 2002.

[3] Y.K. Ho and D.J. Ewins, On Structural Damage Identification with Mode Shapes, Proceedings of COST F3 Conference on System Identification and Structural Health Monitoring, Madrid, Spain, 2000, pp. 677-686.

[4] N. Stubbs and J.T. Kim, Damage Localization in Structures without Baseline Modal Parameters, American Institute of Aeronautics and Astronautics Journal 34(8) (1996), 1649-1654.

[5] M.M.F. Yuen, A Numerical Study of the Eigenparameters of a Damaged Cantilever, Journal of Sound and Vibration 103 (1985), $301-310$.

[6] A.K. Pandey, M. Biswas and M.M. Samman, Damage Detection from Changes in Curvature Mode Shapes, Journal of Sound and Vibration 145(2) (1991), 321-332.

[7] A. Wahab and G. Roeck, Damage Detection in Bridges Using Modal Curvatures: Application to a Real Damage Scenario, Journal of Sound and Vibration 226 (1999), 217-235.

[8] N.M.M Maia, J.M.M. Silva, E.A.M. Almas and R.P.C. Sampaio, Damage Detection in Structures: from Mode Shape to Frequency Response Function Methods, Mechanical Systems and Signal Processing 17(3) (2003), 489-498.

[9] D. Wu and S.S. Law, Damage Localization in Plate Structures from Uniform Load Surface Curvature, Journal of Sound and Vibration 276 (2004), 227-244

[10] C.P. Ratcliffe and W.J. Bagaria, A Vibration Technique for Locating Delamination in a Composite Beam, American Institute of Aeronautics and Astronautics Journal 36(6) (1998), 1074-1077.

[11] M. Gherlone, M. Mattone, C. Surace, A. Tassoti and A. Tessler, Novel Vibration-Based Methods for Detecting Delamination Damage in Composite Plate and Shell Laminates, Key Engineering Materials 293-294 (2005), 289-296. 

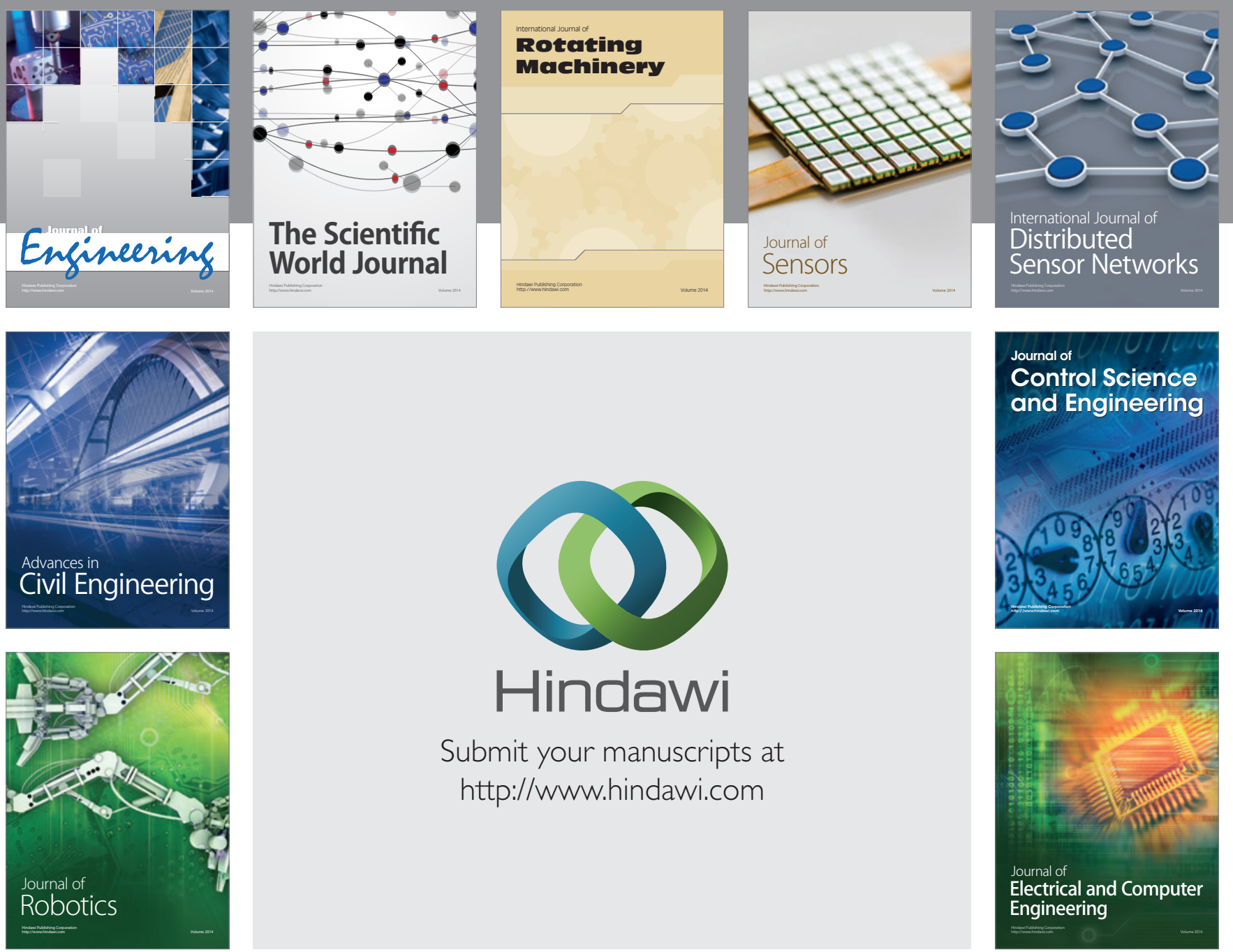

Submit your manuscripts at

http://www.hindawi.com
